\title{
Bipolarization L-band microwave radiometer
}

\author{
Alexandr Gudkov ${ }^{1}$, Vitaly Leushin ${ }^{1,2}$, Svetlana Agasieva ${ }^{3,4}$, and Igor Sidorov ${ }^{4,5, *}$ \\ ${ }^{1}$ Bauman Moscow State Technical University, 105005, 2-ya Baumanskaya st., 5, Moscow, Russia \\ ${ }^{2}$ Open Company "Scientific and Production Company "HIPERION", 121170, Kutuzov ave., 34, \\ Moscow, Russia \\ ${ }^{3}$ RUDN University, 117198, Mikluho-Maclay st. 6, Moscow, Russia \\ ${ }^{4}$ Open Company "Miraglobex", 115201, Kashirskiy pass, 13 room 12, Moscow, Russia \\ ${ }^{5}$ JSC Radio Engineering Corporation "VEGA”, Departmenf of Radar Systems, 121170, Kutuzov ave., \\ 34, Moscow, Russia
}

\begin{abstract}
A new bipolarization L-band microwave radiometer designed to measure soil moisture from an unmanned helicopter is presented. The schematic of the radiometer and an overview of the radiometer parts circuits are considering. The main features of the implementation of circuit and technical solutions are considered. Algorithms of operation of the control and processing microcontrollers are given.
\end{abstract}

\section{Introduction}

The development of precision farming technologies requires the prompt receipt of accurate and complete information about the state of soils to optimize the processes of tillage in the cultivation of crops. Therefore, the task of remote measuring the soil moisture and surface temperature by microwave radiometric sensing of large areas of agricultural land from the Board of an unmanned helicopter is urgent. The solution of the presented problem is possible by means of a portable, light microwave radiometer operating simultaneously on two polarizations, for example, in the L-band. The specifics of construction and application of microwave radiometric systems for remote monitoring of subsurface soil moisture are widely presented in scientific periodicals [1-3]. The purpose of the article is to demonstrate the basic circuit, technical and software solutions for the implementation of a portable Lband microwave radiometer, designed for remote measurement of soil moisture in a layer up to one meter depth from the board of the unmanned helicopter.

Microwave radiometer designed for unmanned helicopter must have small size and weight, low energy consumption and be built on the "all in one" scheme, when the radiometric system except the receiver and antenna includes a satellite navigation receiver, data acquisition system, micro-navigation system and accumulator battery. The descriptions of the some parts of the radiometer system have already been reported at previous conferences [4].

\footnotetext{
* Corresponding author: igorasidorov@yandex.ru
} 


\section{Main part}

The design of the new L-band radiometer consists of two main units - the antenna and the receiver (Fig.1).

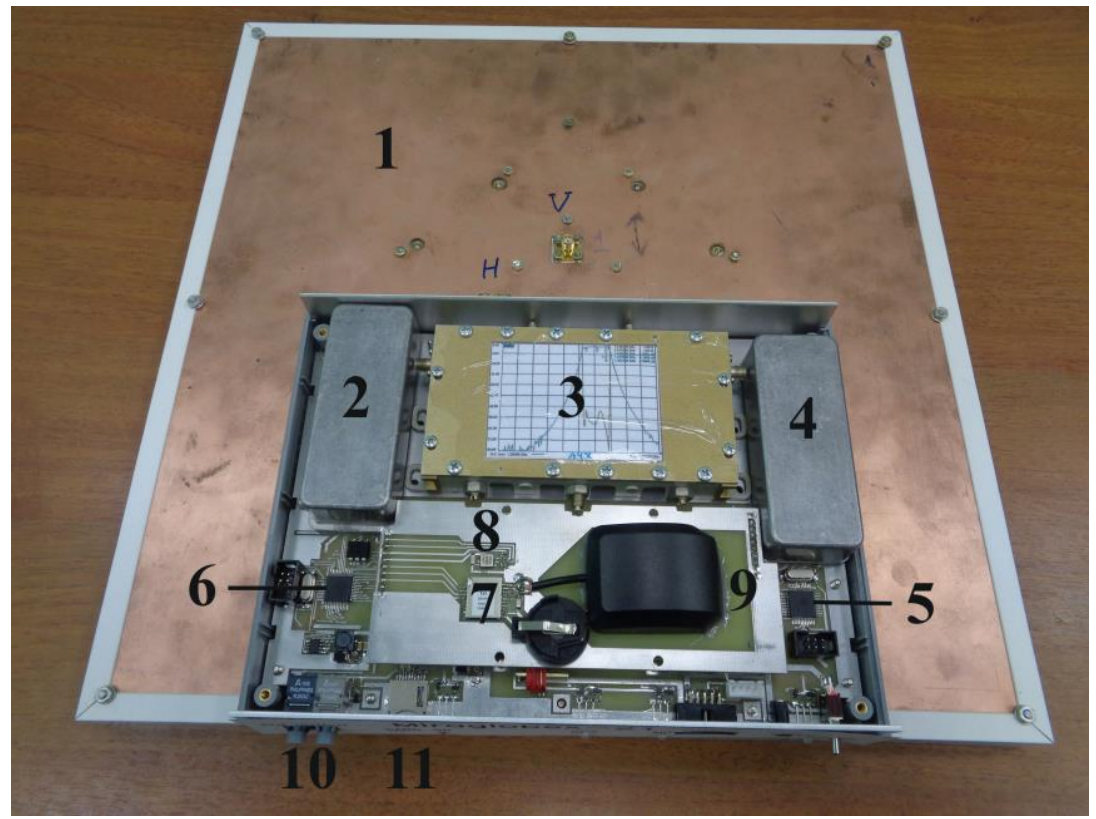

Fig. 1. The design of the new L-band radiometer.

The antenna (1) have two output connectors for vertical and horizontal polarization waves. It connects by coaxial cables with inputs of modulator (2). After the band pass filter (3), the signal is amplified by a high-frequency amplifier and detected by a square detector (4). The signal from the detector output is fed to the ADC input of the signal processing microcontroller (5). After pre-processing, the information is transferred to the control microcontroller (6), which generates an output data stream in a specified format for transmission over the optical interface (10) and recording to the memory card (11). The GPS receiver (7) with GPS antenna (7) transfers the navigation data to the control microcontroller (6). The dual-axis accelerometer (8) measures the rotation and peach angles and send the data to the control microcontroller (6).

The antenna of the radiometer is designed according to the bipolarization scheme, thus the double economy of weight and size. The radiometric receiver is designed according to the scheme with two references modulation, since it does not require to use a thermostat for thermal stabilization of the radiometric path, which provides energy savings and increases the duration of operation from an autonomous source. The band pass filter is selected air type on the counter rods, which provides the best selectivity, although slightly increases the size and weight compared to microstrip filters. Radiometric information processing and control, monitoring and recording tasks should be divided between two microcontrollers.

The radiometer is working on the following algorithm. The control microcontroller (6) is forming the control signals to switch periodically the modulator. So that the first quarter of the modulation period the signal from antenna vertical polarization output supplies to the amplifier input. Then the second quarter of the modulation period the signal from antenna horizontal polarization output supplies to the amplifier input. Then the third quarter of the modulation period the signal from hot reference load supplies to the amplifier input. Then the last quarter of the modulation period the signal from cold reference load supplies to the 
amplifier input. The signal processing microcontroller (5) collecting this data during one second and calculates the antenna temperatures both for vertical and for horizontal polarization by formula (1).

$$
T_{b r}=\frac{U_{A}-U_{C}}{U_{H}-U_{C}} \cdot\left(T_{H}-T_{C}\right)+T_{C}
$$

Were $U_{A}, U_{H}$ and $U_{C}$ are the collected signals from antenna, hot and cold references. $T_{H}$ and $\mathrm{T}_{\mathrm{C}}$ are temperatures of hot and cold references measured by thermal sensors.

\section{Results}

The antenna pattern has been measured on special stend. The measuring results both for Eplane and for H-plane are represented on Fig.2 .
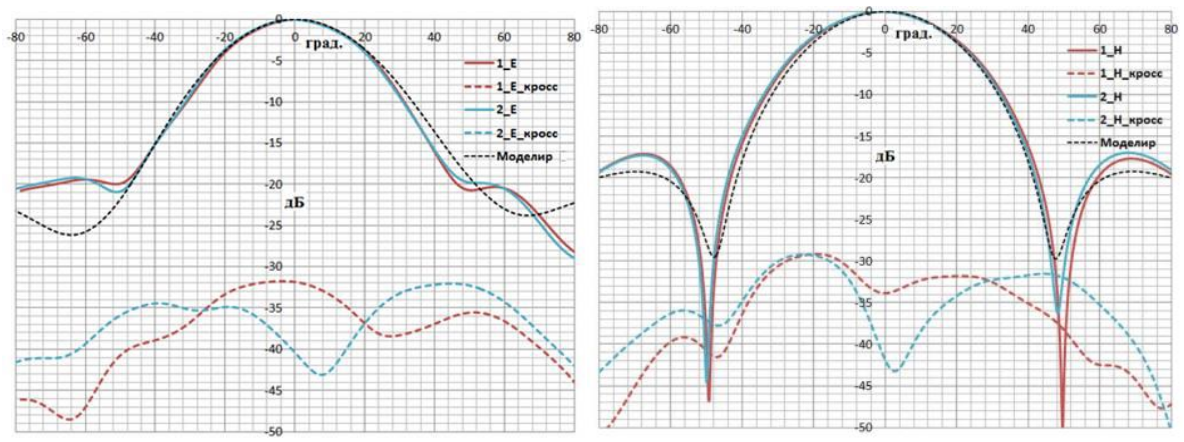

Fig. 2. The antenna patterns for $\mathrm{E}$ and $\mathrm{H}$ planes.

The band pass filter and amplifaer amplitude friquence diagrams are represented on Fig. 3.
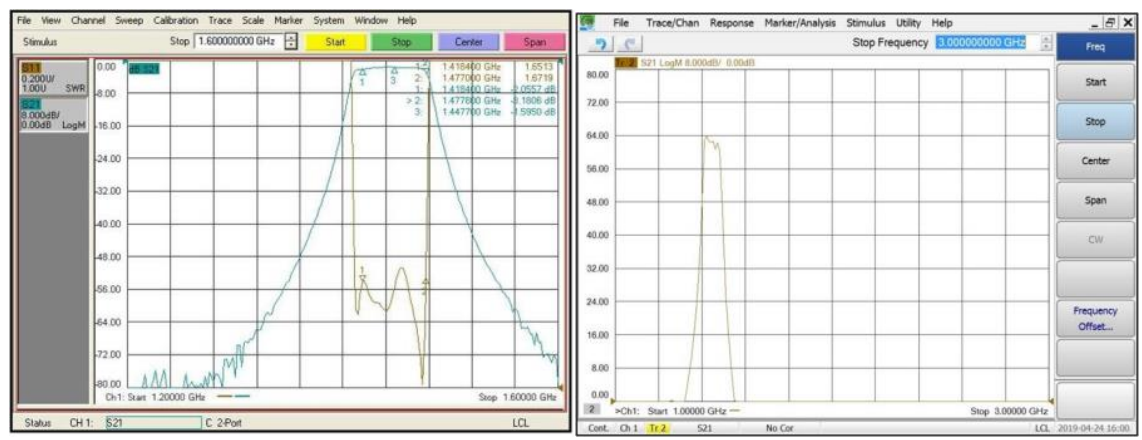

Fig. 3. The band pass filter and amplifaer amplitude friquence diagrams.

The main parameters of the new radiometer are represented in Table 1.

Table 1. The main parameters of the new radiometer.

\begin{tabular}{|l|c|c|c|}
\hline \multicolumn{1}{|c|}{ Parameter title } & Value & Accuracy & Unit \\
\hline Receiver frequency & 1440 & \pm 5 & $\mathrm{MHz}$ \\
\hline Bandwidth on 3db level & 80 & \pm 5 & $\mathrm{MHz}$ \\
\hline Sensitivity on 1 sec. & 0,5 & $\pm 0,1$ & ${ }^{\circ} \mathrm{C}$ \\
\hline
\end{tabular}




\begin{tabular}{|l|c|c|c|}
\hline \multicolumn{1}{|c|}{ Parameter title } & Value & Accuracy & Unit \\
\hline Antenna Weight & 0,65 & $\pm 0,05$ & $\mathrm{Kg}$ \\
\hline Receiver Weight & 1,1 & $\pm 0,05$ & $\mathrm{Kg}$ \\
\hline Antenna Length & 0,36 & $\pm 0,001$ & Meter \\
\hline Antenna Width & 0,36 & $\pm 0,001$ & Meter \\
\hline Antenna Heigth & 0,019 & $\pm 0,001$ & Meter \\
\hline Receiver Length & 0,165 & $\pm 0,001$ & Meter \\
\hline Receiver Width & 0,223 & $\pm 0,001$ & Meter \\
\hline Receiver Heigth & 0,041 & $\pm 0,001$ & Meter \\
\hline
\end{tabular}

\section{Conclusion}

As a result of the research it is possible to make the following conclusions:

- it is possible to develop a portable bipolarization microwave radiometer based on the known scheme with two references sourse modulation;

- joint reception of two polarizations by same antenna allows to reduce the weight of the antenna almost two times at the same size;

- the largest and heaviest element of the radiometer is a band-pass air filter. However, its use is justified by the best radio technical characteristics in comparison with the solidstate microstrip filters.

Research was supported by a grant of the Foundation for the Advancement of Small Businesses in Science and Technology (Innovation Promotion Fund) (Contract No. 2886GS1/45415).

\section{References}

1. I.A. Sidorov, A.P. Soldatenko, A.G. Gudkov, V.Yu. Leushin, E.P. Novichikhin, Results of full-scale experiments on monitoring of hydrological situation along highways by multi-frequency polarimetric system of microwave radiometers. Mashinostroitel, Volume 84, Issue 12, pp. 46-55 ISSN 0025-4568 (2015)

2. A.M. Shutko, E.P. Novichikhin, S.V. Marechek, V.A. Plushev, I.A. Sidorov, E.D. Biryukov, A.D. Reschikov, R. Haarbrink, Polarimetric system of two microwave radiometers "Ranet-21". Radiotechnics. No. 1. pp. 123-126 (2013)

3. I.A. Sidorov, V.A. Plushev, E.P. Novichikhin, A.G. Gudkov, Results of a full-scale experiment on measuring soil moisture using a microwave radiometric receiver from an unmanned aerial vehicle. CriMiCo 2016 - 26th International Crimean Conference Microwave and Telecommunication Technology, Sevastopol, 6-12 September 2016, Volume 10, pp. 2301-2307 (2016)

4. D.A. Gorbachev, A.P. Soldatenko, A.D. Reschikov, N.N. Vlasova, M.I. Sidorova, Design features of the heated reference load for microwave radiometer with tworeferences modulation, CriMiCo 2018 - 28th International Crimean Conference Microwave and Telecommunication Technology, Sevastopol, 9-15 September 2018, Volume 7, pp. 1782-1787 (2018) 